Pacific Journal of Mathematics

PROXIMITY BASES AND SUBBASES 


\section{PROXIMITY BASES AND SUBBASES}

P. L. SHARMA

In this paper we provide a definition of a proximity-base (subbase); this enables us to prove results analogous to those in topological and uniform spaces. For example we prove that the set of all proximities on a set $X$ forms a complete lattice. Another consequence is that a proximity on a set $X$ can be defined as a certain collection of pseudometrics on $X$. A pseudometric approach to proximities is discussed in [4]. Two definitions of a "proximity base" have been given in literature, one by Császár and Mrowka [1] and the other by Njasted [3]. Neither of these definitions is perfectly satisfactory; the first does not determine a unique proximity whereas for the second (i) it is not known whether every proximity has such a base and (ii) a proximity itself is not a base unless it is discrete.

2. Notations and terminology. The terminology used in this paper, with the exception of the definition of a proximity base is same as in [5]. By $J_{m}$ we denote the set of the first $m$ natural numbers. If $x \in X$ then for $\{x\}$ we briefly write $x$. By $P(X)$, we denote the powerset of a set $X$. The collection of all topologies on a set $X$ is ordered by inclusion. The ordering $\mathscr{T}_{1} \geqq \mathscr{T}_{2}$ is expressed by saying that $\mathscr{T}_{1}$ is finer than $\mathscr{T}_{2}$, or that $\mathscr{T}_{2}$ is coarser than $\mathscr{T}_{1}$. For any collection $\left\{\mathscr{T}_{a}: a \in I\right\}$ of topologies on a set $X$, the notations Sup $\left\{\mathscr{T}_{a}: a \in I\right\}$ and Inf $\left\{\mathscr{T}_{a}: a \in I\right\}$ have their usual meanings. If $\left\{\mathscr{T}_{a}: a \in I\right\}$ is a collection of completely regular topologies on a set $X$ then Sup CR $\left\{\mathscr{T}_{a}: a \in I\right\}$ denotes the coarsest completely regular topology on $X$ which is finer than each $\mathscr{F}_{a}$ for $a \in I$. Similarly the notation Inf $\mathrm{CR}\left\{\mathscr{T}_{a}: a \in I\right\}$ stands for the finest completely regular topology on $X$ which is coarser than each $\mathscr{T}_{a}$ for $a \in I$. If $\left\{\mathscr{T}_{a}: a \in I\right\}$ is a collection of completely regular topologies on a set $X$ then

and

(1) $\operatorname{Sup} \operatorname{CR}\left\{\mathscr{T}_{a}: a \in I\right\}=\operatorname{Sup}\left\{\mathscr{T}_{a}: a \in I\right\}$,

(2) Inf $\operatorname{CR}\left\{\mathscr{T}_{a}: a \in I\right\} \subseteq \operatorname{Inf}\left\{\mathscr{T}_{a}: a \in I\right\}$.

The following example shows that the inclusion between the two topologies in (2) above may be proper.

ExAmple. Let $P=I \times I$ where $I$ is the unit interval $[0,1]$. Fix a point $(x, y)$ in $P$. A base for a topology $\mathscr{T}_{(x, y)}$ is described as follows: For each $(u, v) \in P$ such that $(u, v) \neq(x, y)$ the set $\{(u, v)\}$ is open. A set $G$ containing the point $(x, y)$ is open iff $P-G$ is a finite set. It is easily seen that the topological space $\left(P, \mathscr{T}_{(x, y)}\right)$ is 
$T_{4}$ and hence completely regular. Let $\mathscr{T}=\mathscr{T}_{(0,0)} \cap \mathscr{T}_{(1,1)}$. The topological space $(P, \mathscr{T})$ is not even regular because the point $(0,0)$ has no proper $\mathscr{T}$-closed neighbourhood.

We give the definition of a proximity.

Definition. A proximity on a nonempty set $X$ is a binary relation $\delta$ on $P(X)$ satisfying the axioms (P. 1) through (P. 5) given below:

(P. 1) $\quad(\phi, X) \notin \delta$

(P. 2) If $A \cap B \neq \phi$ then $(A, B) \in \delta$

(P. 3) $(A, B) \in \delta$ if $(B, A) \in \delta$

(P. 4) $(A, B \cup C) \in \delta$ iff $(A, B) \in \delta$ or $(A, C) \in \delta$

(P. 5) If $(A, B) \notin \delta$ then there exists a set $E \cong X$ such that $(A, E) \notin \delta$ and $(X-E, B) \notin \delta$.

A proximity $\delta$ on $X$ is separated if $(x, y) \in \delta$ implies $x=y$.

Definition. Let $X$ be a nonempty set. If $\theta_{1}$ and $\theta_{2}$ are two binary relations on $P(X)$, then $\theta_{1} \geqq \theta_{2}$ iff $\theta_{1} \subseteq \theta_{2}$. The ordering $\theta_{1} \geqq \theta_{2}$ is expressed by saying that $\theta_{1}$ is finer than $\theta_{2}$ (or $\theta_{2}$ is coarser than $\left.\theta_{1}\right)$.

If $\delta$ is a proximity on $X$ then $\mathscr{T}(\delta)$ denotes the topology on $X$ induced by $\delta$. The proximity on $X$ induced by a pseudometric $d$ is denoted by $\delta(d)$.

3. Base and subbase for a proximity. Let $X$ be a nonempty set. A proximity-base on $X$ is a binary relation $\mathscr{B}$ on $P(X)$ satisfying the axioms (B. 1) through (B. 5) given below:

(B. 1) $(\dot{\phi}, X) \notin \mathscr{B}$

(B. 2) If $A \cap B \neq \phi$ then $(A, B) \in \mathscr{B}$

(B. 3) $(A, B) \in \mathscr{B}$ iff $(B, A) \in \mathscr{B}$

(B. 4) If $(A, B) \in \mathscr{B}$ and $A \subseteq A^{*}, B \subseteq B^{*}$ then $\left(A^{*}, B^{*}\right) \in \mathscr{B}$

(B. 5) If $(A, B) \notin \mathscr{B}$ then there exists a set $E \subseteq X$ such that $(A, E) \notin \mathscr{B}$ and $(X-E, B) \notin \mathscr{B}$.

A proximity-base $\mathscr{B}$ on a set $X$ is separated if $(\{x\},\{y\}) \in \mathscr{B}$ implies $x=y$ for all $x, y \in X$.

THEOREM 3.1. Let $\mathscr{B}$ be a proximity-base on a set $X$ and let a binary relation $\delta(\mathscr{B})$ on $P(X)$ be defined as follows:

$(A, B) \in \delta(\mathscr{B})$ if, given any finite covers $\left\{A_{i}: i \in J_{m}\right\}$ and $\left\{B_{j}: j \in J_{n}\right\}$ of $A$ and $B$ respectively, then there exists a pair $(i, j) J_{m} \times J_{n}$ such that $\left(A_{i}, B_{j}\right) \in \mathscr{B}$.

Then $\delta(\mathscr{B})$ is the coarsest proximity on $X$ finer than the relation 
$\mathscr{B}$. Moreover, the proximity $\delta(\mathscr{B})$ is separated iff the proximitybase $\mathscr{B}$ is separated.

Proof. It is obvious that $\delta(\mathscr{B}) \geqq \mathscr{B}$. Moreover $\delta(\mathscr{B})$ easily satisfies the proximity axioms (P. 1), (P. 2) and (P. 3). Also, if $(A, B) \in \delta(\mathscr{B})$ then $(A, B \cup C) \in \delta(\mathscr{B})$, because any finite cover of $B \cup C$ is, as well, a finite cover of $B$. Now suppose that $(A, B) \in \delta(\mathscr{B})$ and $(A, C) \in \delta(\mathscr{B})$. Then there exists finite covers

$$
\left\{G_{i}: i \in J_{m}\right\},\left\{H_{j}: j \in J_{n}\right\},\left\{L_{k}: k \in J_{r}\right\}
$$

and $\left\{M_{l}: l \in J_{s}\right\}$ of $A, B, A$ and $C$ respectively such that $\left(G_{i}, H_{j}\right) \in \mathscr{B}$ for any $(i, j) \in J_{m} \times J_{n}$ and $\left(L_{k}, M_{l}\right) \in \mathscr{B}$ for any $(k, l) \in J_{r} \times J_{s}$. Let $S_{(i, k)}=G_{i} \cap L_{k} \quad$ and $\quad H_{n+l}=M_{l}$. Then $\left\{S_{(i, k)}:(i, k) \in J_{m} \times J_{r}\right\} \quad$ and $\left\{H_{p}: p \in J_{n \div s}\right\}$ are finite covers of $A$ and $B \cup C$ respectively. By axiom (B. 4) and the above construction, it is clear that $\left(S_{(i, k)}, H_{p}\right) \notin \mathscr{B}$ for any $(i, k) \in J_{m} \times J_{r}$ and $p \in J_{n+s}$. Therefore $(A, B \cup C) \notin \delta(\mathscr{B})$. Thus $\delta(\mathscr{B})$ satisfies the proximity axiom (P.4) also. Now let $(A, B) \notin \delta(\mathscr{B})$. So there exist finite covers $\left\{A_{i}: i \in J_{m}\right\}$ and $\left\{B_{j}: j \in J_{n}\right\}$ of $A$ and $B$ respectively such that $\left(A_{i}, B_{j}\right) \notin \mathscr{B}$ for any $(i, j) \in J_{m} \times J_{n}$. By axiom (B. 5) we can find a set $E_{i j} \subseteq X$ for each $(i, j) \in J_{m} \times J_{n}$, such that $\left(A_{i}, E_{i j}\right) \in \mathscr{B}$ and $\left(X-E_{i j}, B_{j}\right) \in \mathscr{B}$. Let $E_{j}=\cap\left\{E_{i j}: i \in J_{m}\right\}$ and let $E=\cup\left\{E_{j}: j \in J_{n}\right\}$. Then $\left(A_{i}, E_{j}\right) \notin \mathscr{B}$ for any $(i, j) \in J_{m} \times J_{n}$ and so $(A, E) \in \delta(\mathscr{B})$. Also $X-E=\cap\left\{X-E_{j}: j \in J_{n}\right\}$ and

$$
\left(X-E_{j}\right)=\cup\left\{X-E_{i j}: i \in J_{n}\right\} \text {. }
$$

From this we get $\left(X-E_{j}, B_{j}\right) \notin \delta(\mathscr{B})$. Since $\delta(\mathscr{B})$ satisfies the axiom (P. 4), we obtain $\left(X-E, B_{j}\right) \notin \delta(\mathscr{B})$ for any $j \in J_{n}$ and so $(X-E, B) \notin \delta(\mathscr{B})$ (By (P. 4) again). Thus $\delta(\mathscr{B})$ is a proximity on $X$.

Let $\delta$ be any proximity on $X$ such that $\delta \geqq \mathscr{B}$ and let $(A, B) \in \delta$. If $\left\{A_{i}: i \in J_{m}\right\}$ and $\left\{B_{j}: j \in J_{n}\right\}$ are any finite covers of $A$ and $B$ respectively then there exists a pair $(i, j) \in J_{m} \times J_{n}$ such that $\left(A_{i}, B_{j}\right) \in \delta$. Therefore $\left(A_{i}, B_{j}\right) \in \mathscr{B}$. By definition of $\delta(\mathscr{B})$, we have $(A, B) \in \delta(\mathscr{B})$. This proves that $\delta \geqq \delta(\mathscr{B})$. Hence $\delta(\mathscr{B})$ is the coarsest proximity finer than the relation $\mathscr{B}$. It is obvious that $\delta(\mathscr{B})$ is separated iff $\mathscr{B}$ is separated. The proof of the theorem is now complete.

If $\mathscr{B}$ is a proximity-base on a set $X$ then we say that the proximity $\delta(\mathscr{B})$ is generated by the base $\mathscr{B}$.

Definition. Let $X$ be a nonempty set. A proximity-subbase on $X$ is a binary relation $\mathbf{s}$ on $P(X)$ satisfying the axioms (S. 1) and (S. 2) given below:

(S. 1) $A \cap B \neq \varnothing$ implies $(A, B) \in \mathbf{s}$ 
(S. 2) If $(A, B) \notin \mathbf{s}$ then there exists a set $E \cong X$ such that $(A, E) \notin \mathbf{s}$ and $(X-E, B) \notin \mathbf{s}$.

A proximity-subbase $\mathbf{s}$ on $X$ is separated if $\mathbf{s}$ satisfies the following axiom.

(S. 3) If $x, y$ are two distinct elements of $X$ and $(\{x\},\{y\}) \in \mathbf{s}$ then there exist two subsets $P$ and $\mathrm{Q}$ such that $x \in P, y \in Q$ and either $(P, Q) \notin \mathbf{s}$ or $(Q, P) \notin \mathbf{s}$.

THEOREM 3.2. If $\mathbf{s}$ is a proximity-subbase on a set $X$, then there exists a coarsest proximity $\delta(\mathbf{s})$ on $X$ finer than the relation $\mathbf{s}$. Moreover $\delta(\mathbf{s})$ is separated iff $\mathbf{s}$ is separated.

Proof. Define a binary relation $\mathscr{B}(\mathbf{s})$ on $P(X)$ as follows: both

$(A, B) \in \mathscr{B}(\mathbf{s})$ iff $A \neq \varnothing, B \neq \varnothing$ and for any sets $A^{*} \supseteqq A, B^{*} \supseteqq B$,

$\left(A^{*}, B^{*}\right)$ and $\left(B^{*}, A^{*}\right)$ are elements of $\mathbf{s .}$

Obviously $\mathscr{B}(\mathbf{s}) \geqq \mathbf{s}$. We claim that $\mathscr{B}(\mathbf{s})$ is a proximity-base on $X$. The axioms (B. 1) through (B. 4) are easily satisfied by $\mathscr{B}(\mathbf{s})$. To prove that $\mathscr{B}(\mathbf{s})$ satisfies the axiom (B. 5), suppose $(A, B) \notin \mathscr{B}(\mathbf{s})$. Then two cases arise:

Case I. $A=\varnothing$. Take $E=X$. Then $(A, E)=(\varnothing, X) \notin \mathscr{B}(\mathbf{s})$; and $(X-E, B)=(\varnothing, B) \notin \mathscr{B}(\mathbf{s})$. Similarly, when $B=\varnothing$, but $A \neq \varnothing$, we take $E=\varnothing$ and we are through.

Case II. $A \neq \varnothing, B \neq \varnothing$. There exist sets $A^{*} \supseteqq A$ and $B^{*} \supseteqq B$ such that either $\left(A^{*}, B^{*}\right) \notin \mathbf{s}$ or $\left(B^{*}, A^{*}\right) \notin \mathbf{s}$. If $\left(A^{*}, B^{*}\right) \notin \mathbf{s}$ then by axiom (S. 2), there exists a set $E \cong X$ such that $\left(A^{*}, E\right) \notin \mathbf{s}$ and $\left(X-E, B^{*}\right) \notin \mathbf{s}$. Therefore $(A, E) \notin \mathscr{B}(\mathbf{s})$ and $(X-E, B) \notin \mathscr{B}(\mathbf{s})$. Similar arguement applies when $\left(B^{*}, A^{*}\right) \notin \mathbf{s}$.

Thus $\mathscr{B}(\mathbf{s})$ is a proximity-base on $X$. Let $\delta(\mathbf{s})$ be the proximity generated by $\mathscr{B}(\mathbf{s})$. Then $\delta(\mathbf{s}) \geqq \mathscr{B}(\mathbf{s}) \geqq \mathbf{s}$. Let $\delta$ be any proximity on $X$ such that $\delta \geqq \mathbf{s}$. From the definition of $\mathscr{B}(\mathbf{s})$ it easily follows that $\delta \geqq \mathscr{B}(\mathbf{s})$. So by theorem 3.1 we obtain $\delta \geqq \delta(\mathbf{s})$. Thus $\delta(\mathbf{s})$ is the coarsest proximity on $X$ which is finer than the relation $\mathbf{s}$. Moreover it is obvious that $\mathbf{s}$ is separated iff $\mathscr{B}(\mathbf{s})$ is separated. Hence the theorem is proved.

If $\mathbf{s}$ is a proximity-subbase on a set $X$ then the proximity base $\mathscr{B}(\mathbf{s})$ as defined in the proof of Theorem 3.2 above, is the coarsest proximity base on $X$, which is finer than the relation s. We say that the proximity $\delta(\mathbf{s})$ is generated by the proximity-subbase $\mathbf{s}$. Similarly $\mathscr{B}(\mathbf{s})$ is the proximity-base generated by the subbase $\mathbf{s}$.

THEOREM 3.3. Let $\left\{\delta_{a}: a \in I\right\}$ be a nonempty collection of prox- 
imities on a set $X$. Then there exists a coarsest proximity $\delta$ on $X$ such that $\delta$ is finer than $\delta_{a}$ for each $a \in I$.

Proof. Let $\mathscr{B}=\cap\left\{\delta_{a}: a \in I\right\}$. Then $\mathscr{B}$ is a proximity-base, and so it generates a proximity $\delta(\mathscr{B})$. Obviously $\delta(\mathscr{B})$ fulfills the requirements of the theorem. Hence the theorem is proved.

Notation. If $\left\{\delta_{a}: a \in I\right\}$ is a nonempty collection of proximities on a set $X$ then the coarsest proximity on $X$ finer than each $\delta_{a}$ for $a \in I$ is denoted by $\operatorname{Sup}\left\{\delta_{a}: a \in I\right\}$.

COROLlaRY 3.1. Let $\left\{\delta_{a}: a \in I\right\}$ be a nonempty collection of proximities on a set $X$. Then $\mathscr{T}\left[\operatorname{Sup}\left\{\delta_{a}: a \in I\right\}\right]=\operatorname{Sup}\left\{\mathscr{T}\left(\delta_{a}\right): a \in I\right\}$.

The proof follows from the fact that the finest proximity compatible with $\operatorname{Sup}\left\{\mathscr{T}\left(\delta_{a}\right): a \in I\right\}$ is finer than $\delta_{a}$ for each $a \in I$.

THEOREM 3.4. Let $\left\{\delta_{a}: a \in I\right\}$ be a nonempty collection of proximities on a set $X$. Then there exists a finest proximity $\delta$ on $X$ such that $\delta$ is coarser than $\delta_{a}$ for each $a \in I$.

Proof. Let $\alpha$ be the collection of all proximities on $X$ such that $\delta_{p} \in \alpha$ implies that $\delta_{a} \geqq \delta_{p}$ for each $a \in I$. The collection $\alpha$ is nonempty because the indiscrete proximity on $X$ is a member of it. Let $\delta=\operatorname{Sup}\left\{\delta_{p}: \delta_{p} \in \alpha\right\}$. Now we want to prove that $\delta_{a} \geqq \delta$ for each $a \in I$. So take an $a \in I$ and let $(A, B) \in \delta_{a}$. If $\left\{A_{i}: i \in J_{m}\right\}$ and $\left\{B_{j}: j \in J_{n}\right\}$ are arbitrary finite covers of $A$ and $B$ respectively then there exists a pair $(i, j) \in J_{m} \times J_{n}$ such that $\left(A_{i}, B_{j}\right) \in \delta_{a}$. Therefore for the same pair $(i, j),\left(A_{i}, B_{j}\right) \in \delta_{p}$ for each $\delta_{p} \in \alpha$. So $\left(A_{i}, B_{j}\right) \in \mathscr{B}=\cap\left\{\delta_{p}: \delta_{p} \in \alpha\right\}$ and since $\mathscr{B}$ is a proximity-base for $\delta$, we get, $(A, B) \in \delta$. Therefore $\delta_{a} \geqq \delta$. This is true for each $a \in I$. Moreover $\delta$ is finer than each member of $\alpha$. Thus $\delta$ is the finest proximity on $X$ coarser than each member of the collection $\left\{\delta_{a}: a \in I\right\}$. Hence the theorem is proved.

Notation. If $\left\{\delta_{a}: a \in I\right\}$ is a nonempty collection of proximities on a set $X$ then the finest proximity on $X$ which is coarser than each $\delta_{a}$ for $a \in I$ is denoted by $\operatorname{Inf}\left\{\delta_{a}: a \in I\right\}$.

The following corollary is obvious.

COROLlARY 3.2. If $\left\{\delta_{a}: a \in I\right\}$ is a nonempty collection of proximities on a set $X$, then $\mathscr{T}\left[\operatorname{Inf}\left\{\delta_{a}: a \in I\right\}\right] \cong \operatorname{Inf} \mathrm{CR}\left\{\mathscr{T}\left(\delta_{a}\right): a \in I\right\}$.

The following example shows that the inclusion between the two topologies of Corollary 3.2 can sometimes be proper. 
ExAMPLE. Let $(X, \mathscr{T})$ be a Tychonoff space (completely regular $+T_{1}$ ) which is not locally compact. Let $\left\{\delta_{a}: a \in I\right\}$ be the collection of all proximities on $X$ which are compatible with $\mathscr{T}$. Let $\delta=\operatorname{Inf}\left\{\delta_{a}: a \in I\right\}$ and denote by $\mathscr{T}_{0}$ the topology induced on $X$ by d. Since $\mathscr{T}=\mathscr{T}\left(\delta_{a}\right)$ for each $a \in I$, so $\operatorname{Inf} \mathrm{CR}\left\{\mathscr{T}\left(\delta_{a}\right): a \in I\right\}$ is $\mathscr{T}$ itself. But since $(X, \mathscr{T})$ is not locally compact so $\delta$ cannot be compatible with $\mathscr{T}$. Thus $\mathscr{T}_{0}$ is properly contained in $\mathscr{T}$.

Combining Theorems 3.3 and 3.4 we get the following:

THEOREM 3.5. The collection of all proximities on a nonempty set $X$ forms a complete lattice under the ordering $\geqq$.

From Corollary 3.1 it follows that the collection of all proximities on a set $X$ compatible with a completely regular topology on $X$ forms a complete sup-semilattice.

Moreover if $(X, \mathscr{T})$ is a Tychonoff space, then the collection of all proximities on $X$ compatible with $\mathscr{T}$ forms a complete lattice iff the topology $\mathscr{T}$ is locally compact. Thus we have

THEOREM 3.6. The collection $\mathbf{K}$ of all $T_{2}$-compactifications of a Tychonoff space $(X, \mathscr{T})$ forms a complete sup semi-lattice. The collection $\mathbf{K}$ forms a complete lattice iff the topology $\mathscr{T}$ is locally compact also.

THEOREM 3.7. Let $\left(X, \delta_{1}\right)$ and $\left(Y, \delta_{2}\right)$ be two proximity spaces and let $\mathbf{s}$ be a proximity-subbase for the proximity $\delta_{2}$. A function $f:\left(X, \delta_{1}\right) \rightarrow\left(Y, \delta_{2}\right)$ is p-continuous iff $(A, B) \in \mathbf{s}$ implies $\left(f^{-1}(A)\right.$, $\left.f^{-1}(B)\right) \in \delta_{1}$.

Proof. Let $f$ be $p$-continuous. Suppose $(A, B) \notin \mathbf{s}$. Then $(A, B) \notin \delta_{2}$ and therefore $\left(f^{-1}(A), f^{-1}(B)\right) \in \delta_{1}$.

Now we prove the 'if' part. So assume that, if $(A, B) \notin \mathbf{s}$ then $\left(f^{-1}(A), f^{-1}\left(E^{\dagger}\right) \notin \delta_{1}\right.$. Let $\mathscr{B}(\mathbf{s})$ be the proximity-base on $Y$ generated by the subbase $\mathbf{s}$. Suppose $(A, B) \notin \delta_{2}$. We must show that $\left(f^{-1}(A)\right.$, $\left.f^{-1}(B)\right) € \delta_{1}$. If $A=\varnothing$ or $B=\varnothing$ or $(A, B) \notin \mathbf{s}$ then we are through. Also if there exist two sets, $A^{*}$ and $B^{*}$ such that $A^{*} \supseteqq A$ and $B^{*} \supseteqq B$ and either $\left(A^{*}, B^{*}\right) \in \mathbf{s}$ or $\left(B^{*}, A^{*}\right) \notin \mathbf{s}$ even then we are through. In other words we have shown that if the pair $(A, B)$ is not in $\mathscr{B}(\mathbf{s})$ then $\left(f^{-1}(A), f^{-1}(B)\right) \notin \delta_{1}$. Lastly suppose that $(A, B) \in \mathscr{B}(\mathbf{s})-\delta_{2}$. Then these exist finite covers $\left\{A_{i}: i \in J_{m}\right\},\left\{B_{j}: j \in J_{n}\right\}$ of $A, B$ with $\left(A_{i}, B_{j}\right) \in \mathscr{B}(\mathbf{s})$ for any $(i, j) \in J_{m} \times J_{n}$. This implies that $\left(\bigcup_{1}^{m} f^{-1}\left(A_{i}\right)\right.$, $\left.\bigcup_{1}^{n} f^{-1}\left(B_{j}\right)\right) \notin \delta_{1}$ for any $(i, j) \in J_{m} \times J_{n}$ : and so $\left(f^{-1}(A), f^{-1}(B)\right) \notin \delta_{1}$. Therefore $f$ is $p$-continuous. Hence the theorem is proved. 
THEOREM 3.8. Let $X$ be a nonempty set and let $F$ be a non-void family of functions, each member of $F$ being on $X$ into a proximity space $\left(Y_{f}, \delta_{f}\right)$. Then there exists a coarsest proximity on $X$ such that each member of $F$ is p-continuous.

Proof. Define a binary relation $\mathscr{B}$ on $P(X)$ as follows:

$$
(A, B) \in \mathscr{P} \text { iff }(f(A), f(B)) \in \hat{\delta}_{f} \text { for each } f \in F \text {. }
$$

We claim that $\mathscr{B}$ is a proximity-base on $X$. Only the axiom (B. 5) requires verification. Suppose $(A, B) \notin \mathscr{S}$. Then there exists some $f \in F$ such that $(f(A), f(B)) \notin \delta_{f}$. So there is a set $E_{f} \leqq Y_{f}$ such that $\left(f(A), E_{f}\right) \in \delta_{f}$ and $\left(Y_{f}-E_{f}, f(B)\right) \in \delta_{f}$. Let $E=f^{-1}\left(E_{f}\right)$. Then $(A, E) \notin \mathscr{B}$ and $(X-E, B) \notin \mathscr{B}$. Thus $\mathscr{B}$ is a proximity-base on $X$. The proximity $\delta(\mathscr{B})$ on $X$ generated by the base $\mathscr{B}$ is the required proximity. Hence the theorem is proved.

It is possible to generalise the above theorem by replacing any proximity $\delta_{f}$ by a base $\mathscr{B}_{f}$ of it.

In fact the following stronger form can be proved with the aid of Theorem 3.7.

THEOREM 3.9. Let $X$ be a nonempty set and let $F$ be a non-void family of functions, each member $f$ of $F$ being on $X$ onto a proximity space $\left(Y_{f}, \delta_{f}\right)$. For each $f \in F$, let $\widetilde{S}_{f}$ be a proximity subbase for $\hat{o}_{f}$. Then a proximity subbase $\widetilde{S}$ for the coarsest proximity on $X$ which makes each $f \in F$ p-continuous is defined by:

$$
(A, B) \in \widetilde{S} \quad \text { iff } \quad(f(A), f(B)) \in \widetilde{S}_{f} \text { for each } f \in F .
$$

By making use of proximity-bases (proximity-subbases) several theorems in proximity-spaces can be drastically simplified. For example, the following theorem provides a much simpler definition of the product proximity.

THEOREM 3.10. Let $\left\{\left(X_{a}, \delta_{a}\right): a \in I\right\}$ be a nonempty collection of proximity spaces, and let $Z=I I\left\{X_{a}: a \in I\right\}$. The binary relation $\mathscr{B}$ on $P(Z)$ defined by

$$
(A, B) \in \mathscr{B} \quad \text { iff } \quad\left(P_{a}(A), P_{a}(B)\right) \in \delta_{a} \text { for each projection } P_{a},
$$

is a proximity-base on $Z$ for the product proximity.

The proof follows from Theorem 3.8.

In view of Theorem 3.9, it follows that if $\widetilde{S}_{a}$ is a proximity subbase for $\delta_{a}$ then a proximity-subbase $\widetilde{S}$ for the product proximity on $Z$ can be defined as follows: 


\section{$(A, B) \in \widetilde{S} \quad$ iff $\quad\left(P_{a}(A), P_{a}(B)\right) \in \widetilde{S}_{a} \quad$ for each projection $\quad P_{a}$.}

The analogues of our next theorem are well-known in topological and Uniform spaces.

THEOREM 3.11. Let $\left\{\left(X_{a}, \delta_{a}\right): a \in I\right\}$ be a nonempty collection of proximity spaces and let $\left(Z, \delta_{I I}\right)$ be the product proximity space. A function $f$ on a proximity space $(X, \delta)$ to the product $\left(Z, \delta_{I I}\right)$ is p-continuous iff its composition $P_{a} \circ f$ is p-continuous for each projection $P_{a}$.

Proof. If $f$ is $p$-continuous then $P_{a} \circ f$ is $p$-continuous for each $a \in I$, because each projection $P_{a}$ is $p$-continuous. Now suppose $P_{a} \circ f$ is $p$-continuous for each $a \in I$. Let $\mathscr{B}$ be the proximity-base on $Z$ defined by: $(A, B) \in \mathscr{B}$ iff $\left(P_{a}(A), P_{a}(B)\right) \in \delta_{a}$ for each $a \in I$. Suppose $(A, B) \notin \mathscr{B}$. Then there exists an $a \in I$ such that $\left(P_{a}(A), P_{a}(B)\right) \notin \delta_{a}$. As $P_{a} \circ f$ is $p$-continuous, so $\left(\left(P_{a} \circ f\right)^{-1} P_{a}(A),\left(P_{a} \circ f\right)^{-1} P_{a}(B)\right) \notin \delta$. Moreover $A \subseteq P_{a}^{-1} P_{a}(A)$ and $B \cong P_{a}^{-1} P_{a}(B)$. Therefore $\left(f^{-1}(A), f^{-1}(B)\right) \notin \delta$. Thus by Theorem 3.7. $f$ is p-continuous. Hence the theorem is proved.

THEOREM 3.12. If $\delta$ is any proximity on a set $X$, then there exists a nonempty collection $\left\{\delta_{a}: a \in I\right\}$ of pseudometric proximities on $X$ such that $\delta=\operatorname{Sup}\left\{\delta_{a}: a \in I\right\}$.

Proof. Suppose $(A, B) \notin \delta$. Then there exists a $p$-continuous function $f_{A B}$ on $X$ to $[0,1]$ such that $f_{A B}(A)=0$ and $f_{A B}(B)=1$. Define a pseudometric $d\left(f_{A B}\right)=d$ on $X$ by: $d(x, y)=\left|f_{A B}(x)-f_{A B}(y)\right|$ for all $x, y$ in $X$. Let $\delta(d)$ be the proximity on $X$ induced by $d$. We claim that $\delta \geqq \delta(d)$. Suppose $(P, Q) \notin \delta(d)$. Then $d(P, Q)=\varepsilon$ where $\varepsilon>0$. So, for any $p \in P, q \in Q$ we have $d(p, q) \geqq \varepsilon$. Therefore if $\widetilde{d}_{0}$ is the unique proximity on $[0,1]$ then $(f(P), f(Q)) \notin \widetilde{d}_{0}$ and since $f$ is $p$-continuous so $(P, Q) \notin \delta$.

Thus we have shown that $\delta \geqq \delta(d)$, where $d=d\left(f_{A B}\right)$. It follows that $\delta \geqq \operatorname{Sup}\left\{\delta(d): d=d\left(f_{A B}\right)\right.$ and $\left.(A, B) \notin \delta\right\}$. Moreover, if $(A, B) \notin \delta$ then $(A, B) \notin \delta(d)$ where $d=d\left(f_{A B}\right)$, Thus we get $\delta=\operatorname{Sup}\{\delta(d): d=$ $d\left(f_{A B}\right)$ and $\left.(A, B) \notin \delta\right\}$. Hence the theorem is proved.

The following corollary is now obvious.

Corollary 3.3. Let $\delta$ be a proximity on $X$ and let $D$ be the collection of all pseudometrics on $X$ such that $\delta \geqq \delta(d)$ for each $d \in D$. Then we have

$$
\delta=\cap\{\delta(d): d \in D\}
$$


The analogue of the following theorem is well-known in uniformspaces.

THEOREM 3.13. Every proximity space is proximially-isomorphic to a subspace of a product of pseudometric proximity spaces.

Proof. Let $\delta$ be a proximity on $X$ and let $P$ be the collection of all pseudometrics on $X$ such that $\delta \geqq \delta(d)$ for each $d \in P$. By Corollary 3.3 we have $\delta=\cap\{\delta(d): d \in P\}$. Let $Z=I I\{X: d \in P\}$ and let $f$ be the mapping of $X$ into $Z$ defined by $P_{d}(f(x))=x$ for each $x \in X$ and each projection $P_{d}$. Let $Z$ be given the product proximity $\delta^{*}$ where the $d$ th coordinate space of the product has the proximity $\delta(d)$. The composition of $f$ with each projection is the identity map on $X$. Therefore, by Theorem 3.9 the mapping $f$ on $(X, \delta)$ to $\left(Z, \delta^{*}\right)$ is $p$-continuous. Moreover if $(A, B) \notin \delta$ then there exist $d \in P$ such that $(A, B) \notin \delta(d)$; and so $(f(A), f(B)) \notin \delta^{*}$ by definition of product proximity. Thus $f$ is a proximal isomorphism of $(X, \delta)$ into $\left(Z, \delta^{*}\right)$. Hence the theorem is proved.

A clue to a pseudometric approach to proximities (see [4]) is provided by Theorems 3.12 and 3.13 .

4. Remoteness chains. If $\delta_{1}$ and $\delta_{2}$ are two proximities on a set $X$ such that $\delta_{1} \geqq \delta_{2}$ then $\mathscr{T}\left(\delta_{1}\right) \geqq \mathscr{T}\left(\delta_{2}\right)$. Conversely if $\mathscr{T}_{1}$ and $\mathscr{T}_{2}$ are two completely regular topologies on $X$ such that $\mathscr{T}_{1} \geqq \mathscr{I}_{2}$ and if $\delta_{2}$ is any proximity on $X$ compatible with $\mathscr{T}_{2}$ then there always exists a proximity $\delta_{1}$ on $X$ compatible with $\mathscr{T}_{1}$ such that $\delta_{1} \geqq \delta_{2}$. For example, for $\delta_{1}$ we could take the proximity of functional indistinction for $\mathscr{T}_{1}$. The following problem arises:

Problem $I$. Given $\mathscr{T}_{1}$ and $\mathscr{T}_{2}$ are two completely regular topologies on a set $X$ such that $\mathscr{T}_{1} \geqq \mathscr{F}_{2}$ and also given a proximity $\delta_{1}$ on $X$ compatible with $\mathscr{T}_{1}$. Under what conditions will there exist a proximity $\delta_{2}$ on $X$ compatible with $\mathscr{T}_{2}$ such that $\delta_{1} \geqq \delta_{2}$ ?

The following example shows that such a proximity $\delta_{2}$ need not always exist.

ExAMPLE. Let $R$ be the real line and let $\mathscr{T}_{1}, \mathscr{T}_{2}$ respectively be the discrete topology and the usual topology on $R$. Let $\delta_{1}$ be the coarsest proximity on $R$ compatible with $\mathscr{T}_{1}$. Take two sets $A=[1,2]$ and $B=[3,4]$. Then $(A, B) \in \delta_{1}$ because both $A$ and $B$ are $\mathscr{T}_{1}$-closed and $\mathscr{T}_{1}$-noncompact. Let $\delta_{2}$ be any proximity on $R$ compatible with $\mathscr{T}_{2}$. Since $A$ is $\mathscr{T}_{2}$-compact and both $A$ and $B$ are $\mathscr{T}_{2}$-closed, disjoint sets, so $(A, B) \notin \delta_{2}$. Hence for any proximity $\delta_{2}$ on $R$ compatible with $\mathscr{T}_{2}$, it would be impossible to have $\delta_{1} \geqq \delta_{2}$. 
THEOREM 4.1. Let $\mathscr{T}_{1}$ and $\mathscr{T}_{2}$ are two completely regular topologies on a set $X$ such that $\mathscr{T}_{1} \geqq \mathscr{T}_{2}$. Let $\delta_{1}$ be a proximity on $X$ compatible with $\mathscr{T}_{1}$ and denote by $\delta^{* *}$ the proximity of functional indistinction for $\mathscr{T}_{2}$. A necessary and sufficient condition for the existence of a proximity $\delta_{2}$ on $X$ compatible with $\mathscr{T}_{2}$ and satisfying $\delta_{1} \geqq \delta_{2}$, is that the proximity Inf $\left\{\delta_{1}, \delta^{* *}\right\}$ must be compatible with $\mathscr{T}_{2}$.

Proof. The sufficiency part is obvious. To prove the necessity part suppose there exists a proximity $\delta_{2}$ on $X$ compatible with $\mathscr{T}_{2}$ such that $\delta_{1} \geqq \delta_{2}$. Then we have $\delta^{* *} \geqq \operatorname{Inf}\left\{\delta_{1}, \delta^{* *}\right\} \geqq \delta_{2}$. Since both the proximities $\delta^{* *}$ and $\delta_{2}$ induce the topology $\mathscr{T}_{2}$, so Inf $\left\{\delta_{1}, \delta^{* *}\right\}$ must be compatible with $\mathscr{I}_{2}$. Hence the theorem is proved.

Notation. By $D$ we denote the set of all diadic numbers which lie in the interval $[0,1]$. Explicitly $D=\left\{m .2^{-n}: m\right.$ and $n$ are integers and $\left.0 \leqq m \leqq 2^{n}\right\}$.

Definition. Let $(X, \delta)$ be a proximity space and let $\alpha=\left\{E_{i}: i \in D\right\}$ be a collection of subsets of $X$ such that $\left(X-E_{i}, E_{j}\right) \notin \delta$ for any $i, j \in D$ satisfying $i<j$. Then $\alpha$ is called a $\delta$-remoteness sequence for the pair $\left(X-E_{0}, E_{1}\right)$. Moreover the collection $\alpha^{*}=\{(A, B)$ : either $(A, B) \subseteq\left(X-E_{i}, E_{j}\right)$ or $(B, A) \subseteq\left(X-E_{i}, E_{j}\right)$ for some $i, j \in D$ such that $i<j\}$ is called a $\delta$-remoteness chain for the pair $\left(X-E_{0}, E_{1}\right)$. Sometimes we say that $\alpha^{*}$ is a remoteness chain for $\left(X-E_{0}, E_{1}\right)$ with regard to the proximity $\delta$. If $(X, \delta)$ is a proximity space and $(A, B) \notin \delta$ then at least one $\delta$-remoteness chain for $(A, B)$ will surely exist. This can be shown by first constructing a $\delta$-remoteness sequence for the pair $\left(X-E_{0}, E_{1}\right)=(A, B)$ by applying the proximity axiom (P. 5) successively and then by defining a remoteness chain from the remoteness sequence.

THEOREM 4.2. Let $\mathscr{T}_{1}$ and $\mathscr{T}_{2}$ be two completely regular topologies on a set $X$ such that $\mathscr{T}_{1} \geqq \mathscr{T}_{2}$. Let $\delta_{1}$ be any proximity compatible with $\mathscr{T}_{1}$ and let $\delta^{* *}$ be the proximity of functional indistinction for $\mathscr{T}_{2}$. A necessary and sufficient condition for the proximity $\delta_{2}=\operatorname{Inf}$ $\left\{\delta_{1}, \delta^{* *}\right\}$ to be compatible with $\mathscr{T}_{2}$ is that for each pair $(x, A)$ such that $x \notin \mathscr{T}_{2}$-cl $(A)$ there exists a common remoteness chain $\alpha$ for $(x, A)$ with regard to both the proximities $\delta_{1}$ and $\delta^{* *}$.

Proof. Necessity part: Suppose that $\delta_{2}$ is compatible with $\mathscr{T}_{2}$. Take any pair $(x, A)$ such that $x \notin \mathscr{T}_{2}$ - $\mathrm{Cl}(A)$. Then any $\delta_{2}$-remoteness chain for $(x, A)$ is a common remoteness chain for $(x, A)$ with regard to both the proximities $\delta_{1}$ and $\delta^{* *}$. 
Sufficiency part. Suppose $(x, A)$ is a pair such that $x \notin \mathscr{T}_{2}-\operatorname{cl}(A)$. Then there is a common remoteness chain $\alpha$ for $(x, A)$ with regard to both $\delta_{1}$ and $\delta^{* *}$. If $\hat{o}_{0}$ denotes the indiscrete proximity on $X$, then the collection $\mathscr{B}=\hat{o}_{0}-\alpha$ is a proximity base on $X$ and generates a proximity $\delta(\mathscr{B})$. Moreover we have $\hat{o}_{1} \geqq \hat{o}(\mathscr{B})$ and $\delta^{* *} \geqq \delta(\mathscr{B})$ because $\delta_{1} \geqq \mathscr{B}$ and $\delta^{* *} \geqq \mathscr{B}$. Therefore $\delta_{2} \geqq \delta(\mathscr{B})$. Since $(x, A) \in$ $\delta(\mathscr{B})$ so $(x, A) \notin \delta_{2}$. This can be done for each pair $(x, A)$ such that $x \notin \mathscr{T}_{2}$-cl $(A)$. It follows that $\hat{o}_{2}$ is compatible with $\mathscr{T}_{2}$. Hence the theorem is proved.

A solution to problem $I$ is provided by Theorems 4.1 and 4.2 taken together. A comparison between Corollaries 3.1 and 3.2 raises the following problem:

Problem II. Let $\left\{\delta_{a}: a \in I\right\}$ be a non-void collection of proximities on a set $X$. Under what conditions will the topologies $\mathscr{T}\left[\operatorname{Inf}\left\{\delta_{a}: a \in I\right\}\right]$ and Inf CR $\left\{\mathscr{T}\left(\delta_{a}\right): a \in I\right\}$ be same?

An example has already been given to show that the two topologies can be distinct. The following theorem solves this problem.

THEOREM 4.3. Let $\left\{\delta_{a}: a \in I\right\}$ be a non-void collection of proximities on $a$ set $X$ and let $\mathscr{T}^{*}=\operatorname{Inf} \mathrm{CR}\left\{\mathscr{T}\left(\hat{\delta}_{a}\right): a \in I\right\}$, and denote by $\delta^{*}$ the proximity of functional indistinction for $\mathscr{T}^{*}$. A necessary and sufficient condition for the proximity $\hat{o}=\operatorname{Inf}\left\{\delta_{a}: a \in I\right\}$ to induce the topology $\mathscr{T}^{*}$ is that for each pair $(x, A)$ such that $x \notin \mathscr{T}^{*}$-cl $A$, there is a $\delta^{*}$-remoteness chain $\alpha$ for $(x, A)$ so that $\alpha$ is also a remoteness chain for $(x, A)$ with regard to $\hat{o}_{a}$ for each $a \in I$.

The proof of this theorem is similar to those of Theorems 4.1 and 4.2, and is thus omitted.

Several interesting problems on proximities can be solved by making use of proximity bases (subbases).

The author wishes to thank Professor S. A. Naimpally for helpful suggestions.

\section{REFERENCES}

1. Császár and Mrówka, Sur la compactification des espaces proximite, Fund. Math., 46, (1959), 195-207.

2. J. R. Isbell, Uniform space, Providence R. I. (1964).

3. O. Njastad, On Wallman type compactifications, Math. Z., 91, (1966), 267-276.

4. P. L. Sharma and S. A. Naimpally, A pseudometric approach to Proximities, (Sent for Publication.) 
5. S. A. Naimpally and B. D. Narrack, Proximity spaces, Cambridge Tract No. 59, (1970), (In Press).

6. S. Leader, On Products of proximity spaces, Math. Annalen, 154, (1964), 185-194. 7. W. J. Thron, Topological Structures, Holt, Rinehart and Winston.

Received November 30, 1970.

Indian Institute of TechNology KanPUR, India 


\section{PACIFIC JOURNAL OF MATHEMATICS}

\section{EDITORS}

\author{
H. SAMElson \\ Stanford University \\ Stanford, California 94305 \\ C. R. HOBBY \\ University of Washington \\ Seattle, Washington 98105
}

J. DugundjI

Department of Mathematics University of Southern California Los Angeles, California 90007

RICHARD ARENS

University of California

Los Angeles, California 90024

\section{ASSOCIATE EDITORS}
E. F. BECKENBACH
B. H. NeUmanN
F. WOLE
K. YOSHIDA

\section{SUPPORTING INSTITUTIONS}

\author{
UNIVERSITY OF BRITISH COLUMBIA \\ CALIFORNIA INSTITUTE OF TECHNOLOGY \\ UNIVERSITY OF CALIFORNIA \\ MONTANA STATE UNIVERSITY \\ UNIVERSITY OF NEVADA \\ NEW MEXICO STATE UNIVERSITY \\ OREGON STATE UNIVERSITY \\ UNIVERSITY OF OREGON \\ OSAKA UNIVERSITY \\ UNIVERSITY OF SOUTHERN CALIFORNIA
}

\author{
STANFORD UNIVERSITY \\ UNIVERSITY OF TOKYO \\ UNIVERSITY OF UTAH \\ WASHINGTON STATE UNIVERSITY \\ UNIVERSITY OF WASHINGTON \\ * * * \\ AMERICAN MATHEMATICAL SOCIETY \\ CHEVRON RESEARCH CORPORATION \\ NAVAL WEAPONS CENTER
}

The Supporting Institutions listed above contribute to the cost of publication of this Journal, but they are not owners or publishers and have no responsibility for its content or policies.

Mathematical papers intended for publication in the Pacific Journal of Mathematics should be in typed form or offset-reproduced, (not dittoed), double spaced with large margins. Underline Greek letters in red, German in green, and script in blue. The first paragraph or two must be capable of being used separately as a synopsis of the entire paper. The editorial "we" must not be used in the synopsis, and items of the bibliography should not be cited there unless absolutely necessary, in which case they must be identified by author and Journal, rather than by item number. Manuscripts, in duplicate if possible, may be sent to any one of the four editors. Please classify according to the scheme of Math. Rev. Index to Vol. 39. All other communications to the editors should be addressed to the managing editor, Richard Arens, University of California, Los Angeles, California, 90024.

50 reprints are provided free for each article; additional copies may be obtained at cost in multiples of 50 .

The Pacific Journal of Mathematics is published monthly. Effective with Volume 16 the price per volume (3 numbers) is $\$ 8.00$; single issues, $\$ 3.00$. Special price for current issues to individual faculty members of supporting institutions and to individual members of the American Mathematical Society: $\$ 4.00$ per volume; single issues $\$ 1.50$. Back numbers are available.

Subscriptions, orders for back numbers, and changes of address should be sent to Pacific Journal of Mathematics, 103 Highland Boulevard, Berkeley, California, 94708.

PUBLISHED BY PACIFIC JOURNAL OF MATHEMATICS, A NON-PROFIT CORPORATION

Printed at Kokusai Bunken Insatsusha (International Academic Printing Co., Ltd.), 7-17, Fujimi 2-chome, Chiyoda-ku, Tokyo, Japan. 


\section{Pacific Journal of Mathematics}

\section{Vol. 37, No. $2 \quad$ February, 1971}

Charles Compton Alexander, Semi-developable spaces and quotient images of metric spaces .................................... 277

Ram Prakash Bambah and Alan C. Woods, On a problem of Danzer. . . . . . . . . 295

John A. Beekman and Ralph A. Kallman, Gaussian Markov expectations and related integral equations . ....................................

Frank Michael Cholewinski and Deborah Tepper Haimo, Inversion of the Hankel

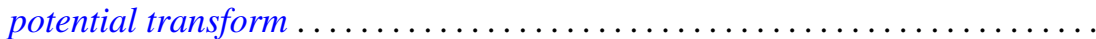

John H. E. Cohn, The diophantine equation

$$
Y(Y+1)(Y+2)(Y+3)=2 X(X+1)(X+2)(X+3) \ldots \ldots \ldots \ldots \ldots
$$

Philip C. Curtis, Jr. and Henrik Stetkaer, A factorization theorem for analytic

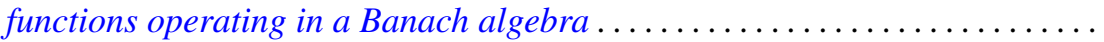

Doyle Otis Cutler and Paul F. Dubois, Generalized final rank for arbitrary limit ordinals

Keith A. Ekblaw, The functions of bounded index as a subspace of a space of entire functions

Dennis Michael Girard, The asymptotic behavior of norms of powers of

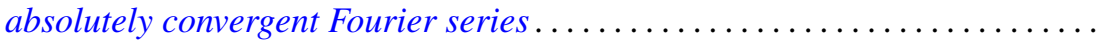

John Gregory, An approximation theory for elliptic quadratic forms on Hilbert spaces: Application to the eigenvalue problem for compact quadratic forms. 383

Paul C. Kainen, Universal coefficient theorems for generalized homology and stable cohomotopy.

Aldo Joram Lazar and James Ronald Retherford, Nuclear spaces, Schauder bases, and Choquet simplexes.

David Lowell Lovelady, Algebraic structure for a set of nonlinear integral operations

John McDonald, Compact convex sets with the equal support property . 429

Forrest Miller, Quasivector topologies

Marion Edward Moore and Arthur Steger, Some results on completability in commutative rings.

A. P. Morse, Taylor's theorem

Richard E. Phillips, Derek J. S. Robinson and James Edward Roseblade, Maximal subgroups and chief factors of certain generalized soluble groups.

Doron Ravdin, On extensions of homeomorphisms to homeomorphisms ...

John William Rosenthal, Relations not determining the structure of $\mathrm{L}$

Prem Lal Sharma, Proximity bases and subbases ........... .

Larry Smith, On ideals in $\Omega_{*}^{u}$. .

Warren R. Wogen, von Neumann algebras generated by operators similar to normal operators

R. Grant Woods, Co-absolutes of remainders of Stone-Čech 